\title{
Observation of flexural cracks in loaded concrete beams using MRI
}

\author{
E. Marfisi*, C. J. Burgoyne*, M. H. G. Amin $†$ and L. D. Hall† \\ University of Cambridge
}

This paper describes apparatus that enables loading of a concrete beam reinforced with aramid fibre-reinforced plastic (AFRP) inside the magnet of a magnetic resonance imaging (MRI) scanner, and the subsequent measurement in three dimensions of the propagation of fractures within the concrete. These are then correlated with the internal structure of the concrete which had been previously determined by scanning the beam soon after casting. The particular requirements of the test frame for use within the scanner and the methods used to satisfy them are described in detail, as is the test procedure. It is shown that the resultant images can be visualised either as twodimensional (2-D) slices, or as 3-D data sets; the images can also be post-processed to highlight the particular feature of interest.

\section{Introduction}

In two companion papers, ${ }^{1,2}$ methods have been developed that allow the internal structure of concrete to be measured using magnetic resonance imaging (MRI), and which show how internal cracks can be observed, measured and correlated with the original structure. Since the fractured samples studied in these papers had been loaded outside the MRI scanner, the measurements were of the cracked but unloaded state. This paper describes hardware that has been developed so that these scanning methods can be used to follow the development of cracks in a concrete beam while it is loaded in flexure inside the MRI scanner.

The experiment was designed to satisfy the following constraints.

(a) The concrete cannot be reinforced with steel since this affects the MRI scanner's magnetic field and hence distorts the resultant magnetic resonance (MR) image.

\footnotetext{
* Department of Engineering, University of Cambridge, Trumpington St, Cambridge CB2 1PZ, UK.

$\dagger$ Herschel Smith Laboratory for Medicinal Chemistry (HSLMC), University of Cambridge School of Clinical Medicine, Robinson Way, Cambridge CB2 2PZ, UK.
}

(MCR 31230) Paper received 5 January 2004; last revised 22 July 2004; accepted 9 September 2004 (b) For the same reason, the aggregate in the concrete must contain no paramagnetic elements; however, white Portland cement (WPC), limestone and silica sand can be used. ${ }^{1}$

(c) None of the equipment inserted in the bore of the magnet can contain any iron or other magnetic materials.

(d) The overall dimensions of the sample are limited. The magnet has a cylindrical bore $310 \mathrm{~mm}$ in diameter and $741 \mathrm{~mm}$ long, inside which is placed the cylindrical gradient coil set (central hole $108 \mathrm{~mm}$ in diameter and $548 \mathrm{~mm}$ long) (Fig. 1). This generates the magnetic fields which allow measurements to be made at different positions within the overall field-of-view.

(e) The cylindrical radio-frequency (RF) coil (central bore $54 \mathrm{~mm}$ in diameter and $163 \mathrm{~mm}$ long) is placed inside the gradient coil cylinder.

( $f$ ) The test beam must be immersed in a water bath when loaded to ensure that all cracks are filled with water. The sample, water bath, loading system and reaction frame must all be located inside this gradient coil, and in or around the RF probe.

( $g$ ) The length of the receiver in the RF probe is $100 \mathrm{~mm}$. The field-of-view (in which accurate measurements can be made), was chosen to be a cube with sides of $70 \mathrm{~mm}$.

(h) The region of the beam to be studied must lie inside this field-of-view throughout the loading process, taking account of the deflections of both the beam and loading frame. 


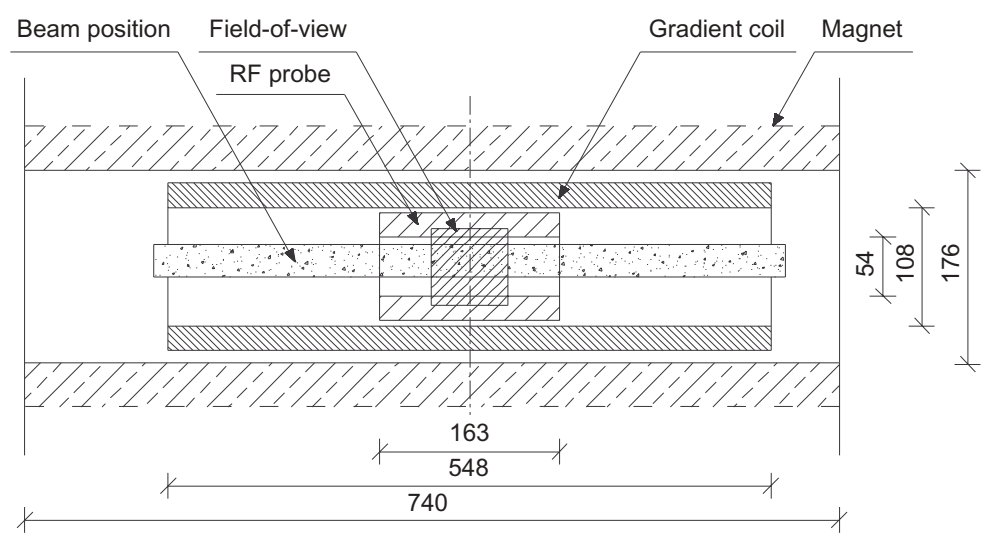

(a)

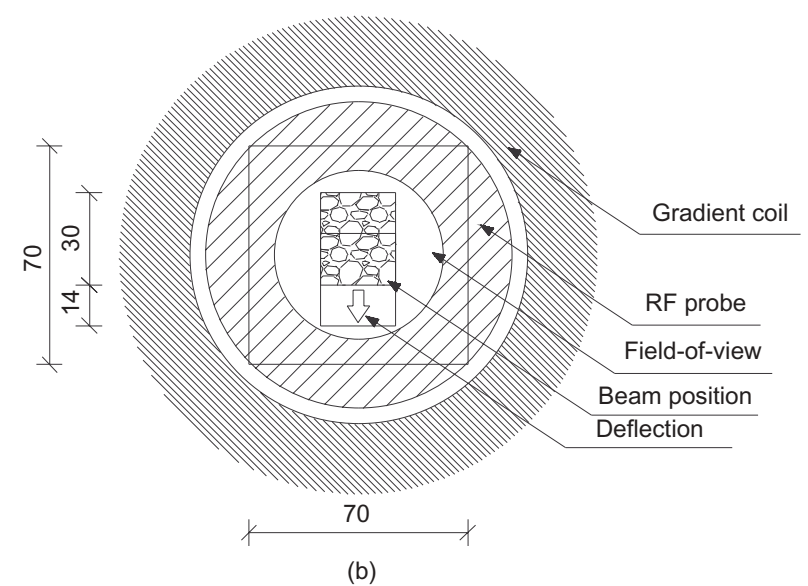

Fig. 1. Internal components of the MRI scanner, showing space available for the test beam: (a) longitudinal section; and (b) cross-section. All dimensions in $\mathrm{mm}$

(i) The beam should not fail catastrophically during the study to avoid damaging the RF probe.

\section{Experimental apparatus}

The test beam

It was decided to perform a flexural bending test under symmetrical loading since this would give a constant moment region in the centre of the beam, part of which would be scanned. The symmetrical arrangement also simplified the design of the reaction frame, since it did not need to carry shear through the most spatially restricted part of the scanner (the RF coil). The loading system could also be located outside the field-of-view.

The chosen beam was $570 \mathrm{~mm}$ long, $30 \mathrm{~mm}$ deep and $25 \mathrm{~mm}$ wide, and was reinforced with bars of aramid fibre-reinforced polymers (AFRP) manufactured from Kevlar 49 aramid yarns, each of cross-sectional area $0.113 \mathrm{~mm}^{2}$. Eight yarns were drawn through a bath of epoxy resin and lightly twisted together to form a bar. Excess resin was removed and the bar kept under light tension while the epoxy hardened. Tests showed that eight-yarn bars had a tensile strength of $2380 \mathrm{MPa}$ and a modulus of $120 \mathrm{GPa}$. The strength conversion efficiency from yarns to bars of $82 \%$ is typical of what can be achieved by hand. With machine-made pultrusions, higher efficiencies can be achieved.

The beam was designed to be slender in order to reach failure by bending within the field-of-view, so shear was not expected to cause failure. Nevertheless, shear links were provided in the form of two-yarn elements that were wrapped around the longitudinal bars and fixed with cyanoacrylate adhesive. The shear links were not tested in tension since their properties are known to be governed by corner effects. ${ }^{3}$

The AFRP reinforcement was then assembled into a cage, as shown in Fig. 2, with two bars on the tension face and two others on the compression face, primarily to give a rigid cage. The shear link spacing was $20 \mathrm{~mm}$ throughout, with inclined stirrups over the loading region.

The concrete mix used sieved white limestone aggregate of various sizes, white cement and deionised water, as shown in Table 1. This mix had been found to be satisfactory in earlier tests using MRI. The effective cube strength of this mix, on the scale used in these experiments ${ }^{4}$ was about $30 \mathrm{MPa}$.

AFRP reinforcement has a virtually linear stressstrain curve to failure. The ultimate moment capacity is governed by the strength of the FRP and was calculated to be $93 \mathrm{Nm}$.

Magazine of Concrete Research, 2005, 57, No. 4 

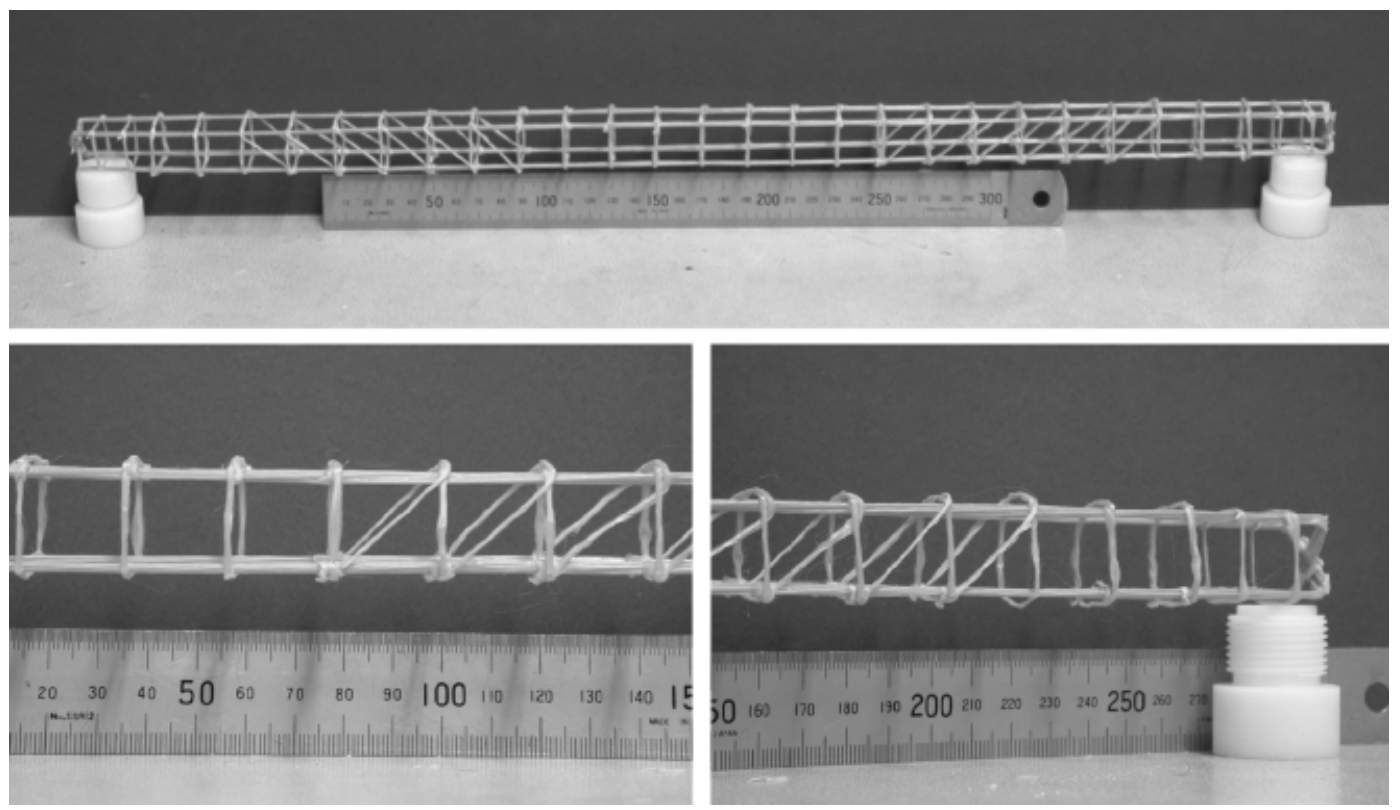

Fig. 2. Details of AFRP beam reinforcement

Table 1. Concrete mix design

\begin{tabular}{l|c|c}
\hline Material & Size: $\mathrm{mm}$ & $\%$ by weight \\
\hline Limestone & $2 \cdot 36-5 \cdot 00$ & 32 \\
& $1 \cdot 70-2 \cdot 36$ & 15 \\
White cement (WPC) & $1 \cdot 40-1 \cdot 70$ & 13 \\
Deionised water & - & 25 \\
\hline
\end{tabular}

\section{The loading system}

The loading system was designed to apply loads to the beam which was installed inside the MRI scanner, under external control. It was found that disposable plastic medical syringes could be used in reverse as jacks, with water as the hydraulic fluid. Fluid pressures up to about $1.2 \mathrm{MPa}$ could be achieved before failure of the sealing gasket between the piston and the cylinder; a $9.5 \mathrm{~mm}$ diameter syringe could thus apply a force of about $400 \mathrm{~N}$.

Since failure of the specimen inside the RF probe had to be avoided, it was decided to load only to $90 \%$ of the moment capacity of the beam, and to reduce the pressure inside the hydraulic system it was decided to use three pistons per side (Fig. 3) at a maximum working pressure of 0.60 $\mathrm{MPa}$.

The outer cylinders were placed in a block of acrylic (PMMA) to avoid problems that would be caused by

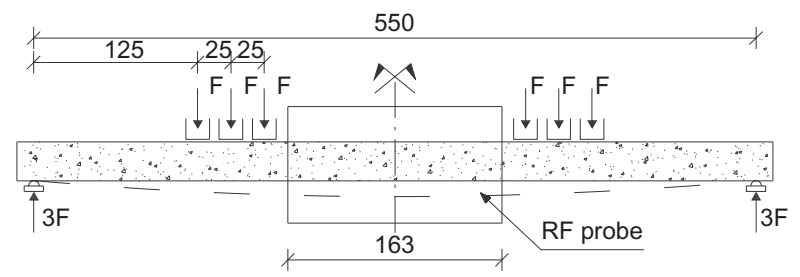

Fig. 3. Beam loading positions (in $\mathrm{mm}$ ) the cylinder swelling under high pressure, and the shaft of the piston was replaced by an acrylic cylinder to avoid buckling. The three cylinders were linked together within the acrylic block so that they all exerted the same pressure and only one connection to external piping was needed. The seals on this external connection proved to be the weakest link in the system.

The displacement was the governing factor during the experiment, with a limit of about $12 \mathrm{~mm}$ on the beam deflection.

\section{The reaction frame}

The supporting frame for the equipment inside the magnet had to be entirely self-reacting, since no contact could be allowed with the magnet or the various coils. The tightest constraint on space was inside the RF probe where the reaction frame had to carry a moment equal and opposite to that carried by the beam. This was achieved by means of polytetrafluoroethylene (PTFE) compression beams that passed inside the RF probe, and tension members made from twisted aramid yarns that were placed in the small space between the RF probe and the gradient coil (Figs 4 and 5). This system had the disadvantage that the RF probe was effectively built-in as part of the reaction frame.

More space was available beyond the ends of the RF probe and the inside of the gradient coil. Acrylic side

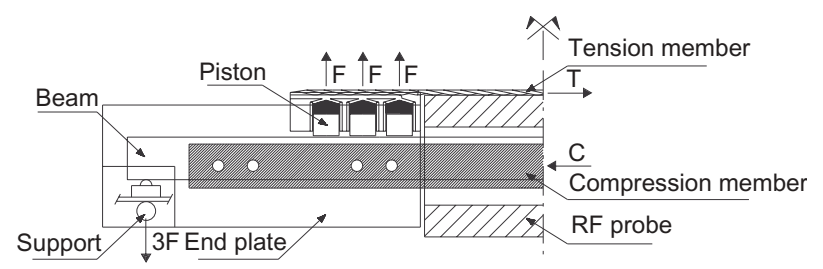

Fig. 4. End details of loading frame 


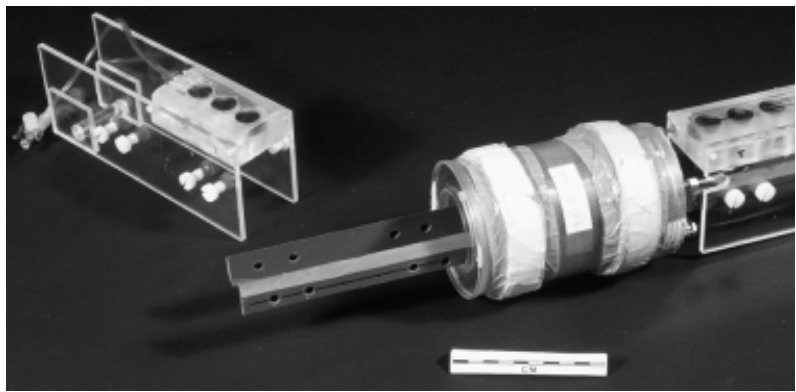

Fig. 5. Loading frame with one end plate and tension members removed

plates supported the cylinder blocks for the loading system and the support bearing for the beam, which was a rod also made from acrylic. The plates were attached to the compression beams by means of nylon bolts. The aramid tension members were loops attached to the cylinder blocks after assembly; they were tensioned by twisting the loops and securing them by means of a nylon rod as shown in Fig. 6.

\section{Saturation system}

In order to ensure the filling of all induced cracks with water, it was necessary for the specimen to be loaded while it was surrounded with water. However, effective MRI measurements could not be taken in that state since the strong signal from the surrounding water would mask the relatively weak signal from the small cracks. Thus the beam was placed in an acrylic water tank which was connected to an external tank by flexible piping. Filling and draining was then achieved by altering the level of the external tank.

The saturation tank had to contain the beam yet fit inside the reaction frame. The beam support bearings thus had to be on the base of the tank, which had to be aligned so that they were immediately above the supporting rod in the reaction frame.

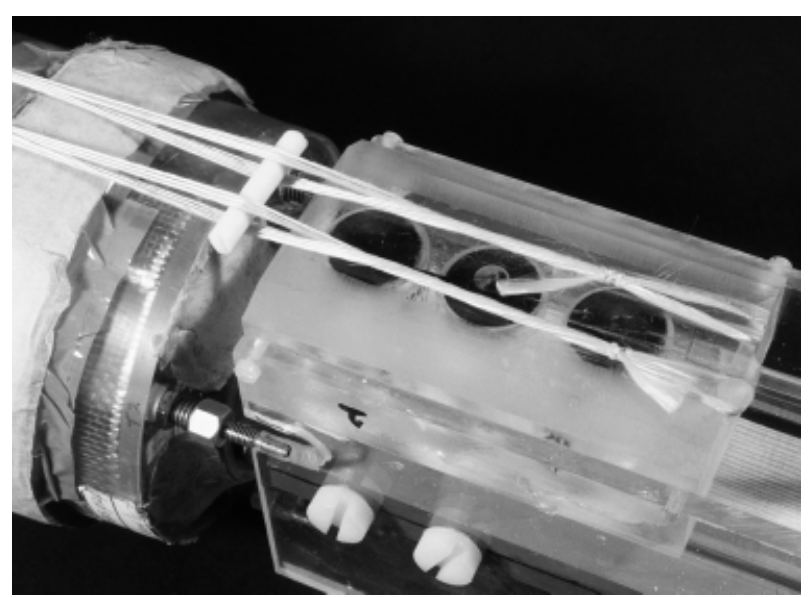

Fig. 6. System for prestressing the tension element

\section{External loading system}

The six hydraulic loading cylinders were arranged in two banks of three, one on each side of the RF probe. For the present test, which was designed to be symmetrical, the two banks of cylinders were connected to a single larger external polythene piston $(28 \mathrm{~mm}$ in diameter) that was loaded by concrete dead weights through a wooden lever system. An adjustable stop ensured that the deflection of the beam remained sensibly constant while the MRI readings were being taken, thus avoiding problems caused by continuing movement of the specimen due to creep of either the concrete or the acrylic loading frame.

\section{Assembly of the test frame}

The reaction frame was assembled with the RF probe in place (Fig. 7). The internal water tank, with the test beam in position, was then inserted and the tension members tightened. The whole assembly could then be placed in the gradient coils (Fig. 8). After the first scan, the loading system was adjusted to bring the pistons into contact with the beam.

Figure 9 shows cross-sections through the assembly at the two critical sections; in the centre of the RF probe and through the loading pistons. The space constraints are clearly visible.

\section{Test procedure}

Previous studies ${ }^{1}$ had shown that the internal structure of concrete can be measured by MRI during the first $10 \mathrm{~h}$ after casting. A set of scans was taken immediately after casting, which did not need either the loading system or the tank in place, so it was relatively easy to reposition the beam inside the scanner. The whole beam was scanned in $70 \mathrm{~mm}$ lengths with about a $20 \mathrm{~mm}$ overlap.

The concrete in the beam was cured under water for four weeks in the laboratory. The beam and test rig were then assembled and placed into the scanner as described above. Before loading, a scan of the central region was carried out to detect any pre-existing fractures or voids.

The beam was loaded monotonically, and the following procedure was adopted for each set of readings.

(a) The beam was submerged by filling the external tank with water.

(b) The chosen load was applied by adding concrete weights to the external loading system.

(c) The adjustable loading stop was used to fix the displacement of the load, which prevented any further displacement of the beam.

(d) The external tank was drained to remove the surrounding water.

(e) The MRI scan was carried out, taking about $7 \mathrm{~h}$. 


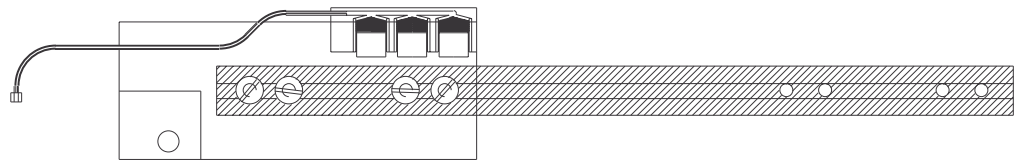

(a)

Connections of the compression members
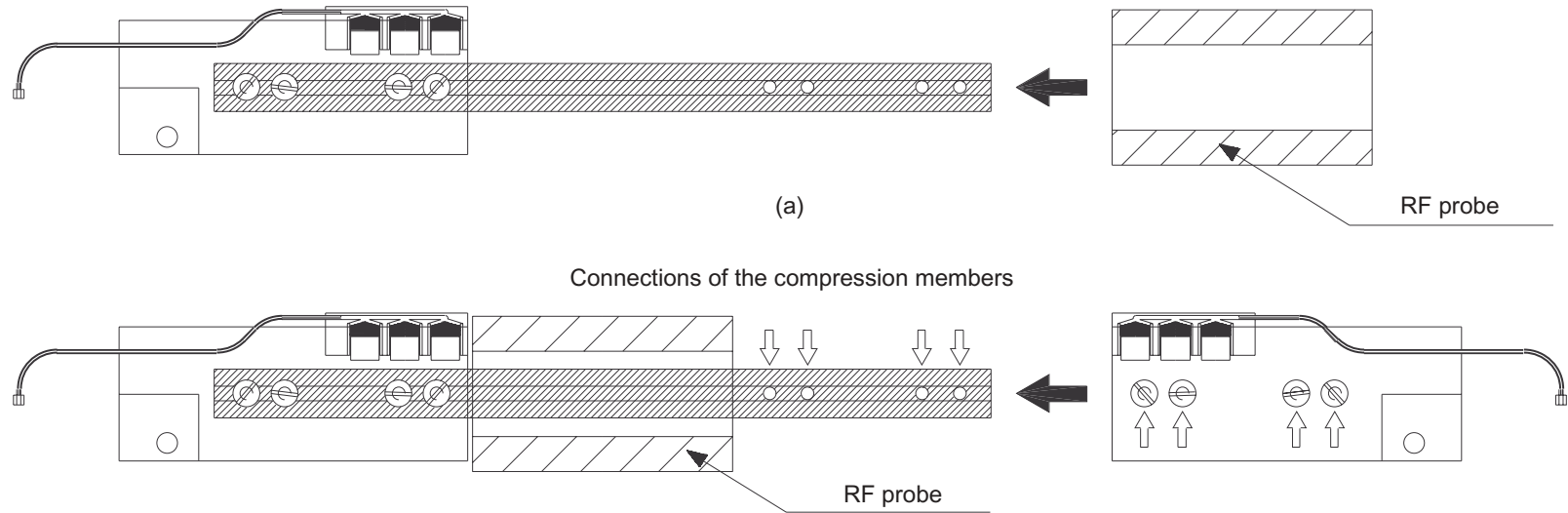

(b)

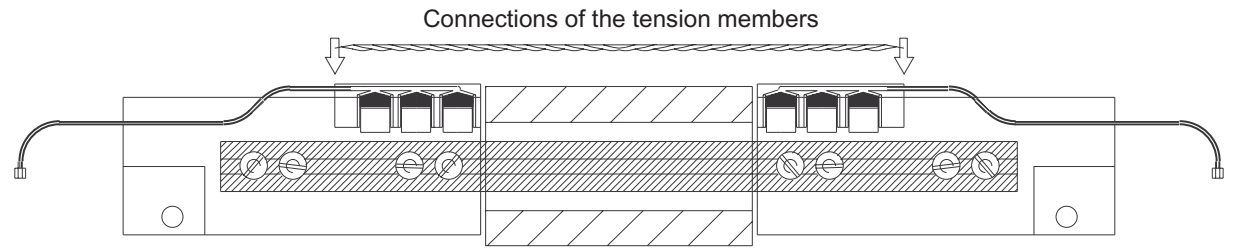

(c)

Fig. 7. Assembly of test rig: (a) installation of RF probe; (b) connection of second end fitting; and (c) connection of tension members

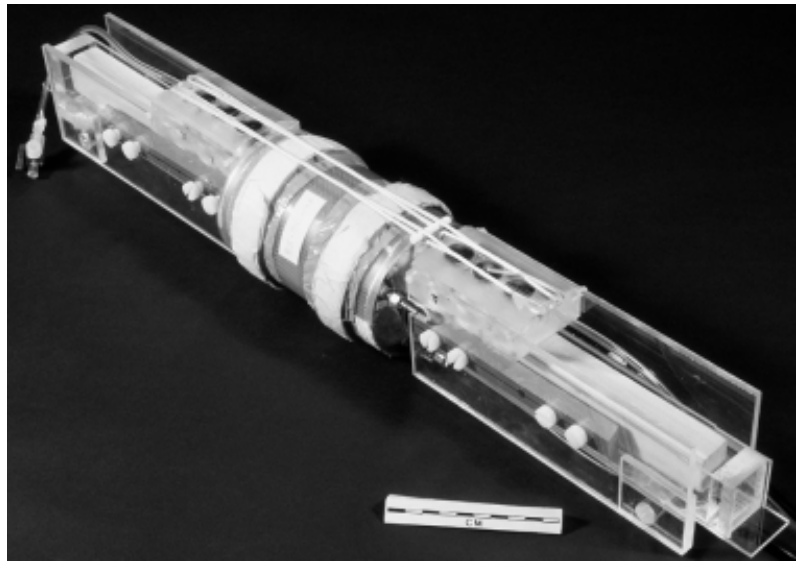

Fig. 8. Assembled frame ready for installation in the $M R$ magnet

The data were then archived and processed after scanning.

Because the complete experiment would take several days, and it would be impossible to adjust the specimen once it was inside the MRI scanner, the entire process was tried out on a second beam outside the scanner so that potential problems could be identified before the real test was carried out. No significant alterations were required.

Magazine of Concrete Research, 2005, 57, No. 4

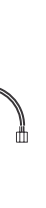

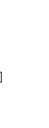


Marfisi et al.

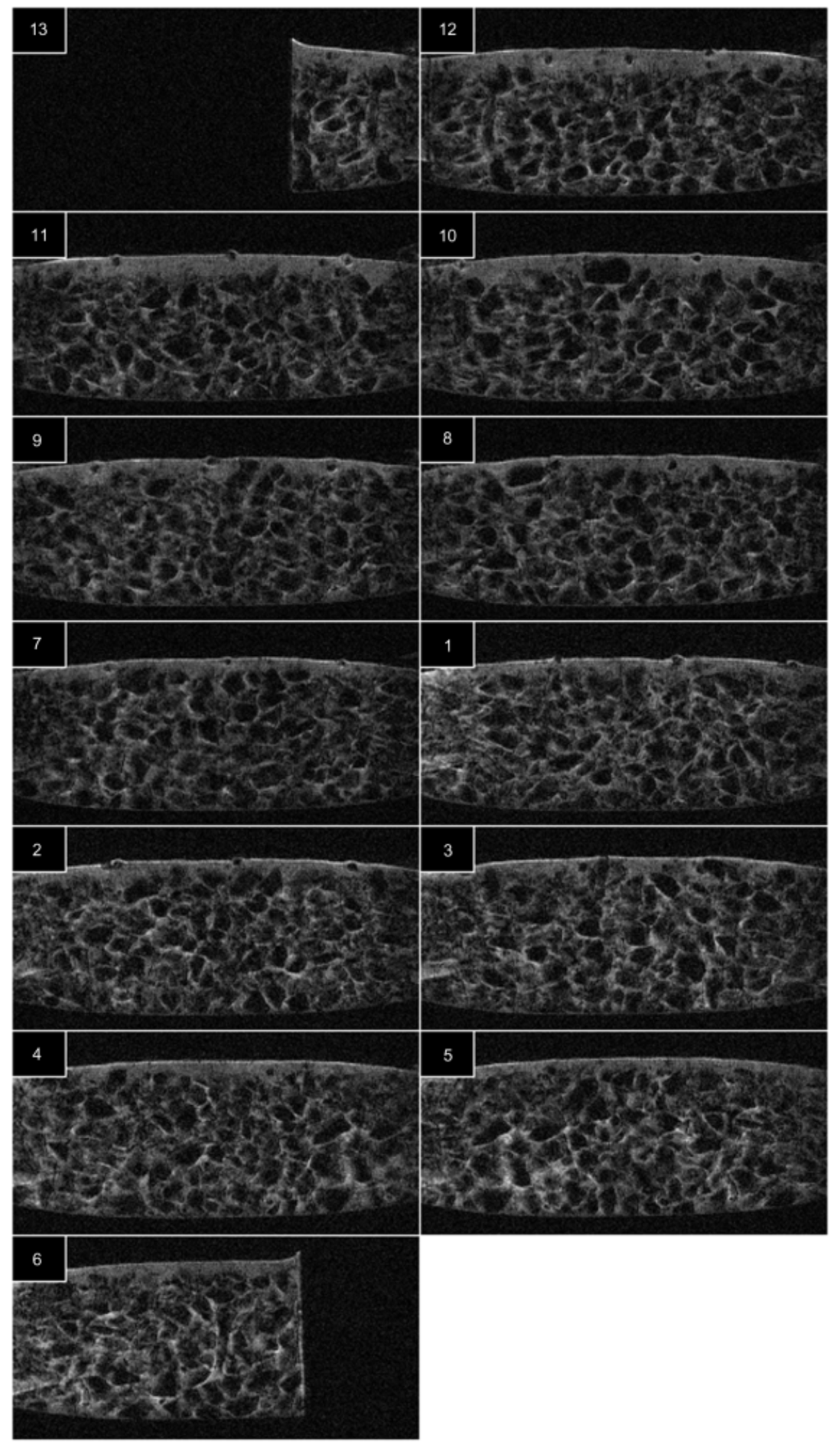

Fig. 10. Beam structure acquisition. The images are in order from top left along the beam. The numbers represent the sequence in which the images were acquired

shown by the number next to each image. This sequence was chosen to get the best image from the central region of the beam which would be scanned during the subsequent loading sequence. The reduction in contrast that took place from the first image to the last is clear in the figure. This reflects the reduction in free water present in the concrete as hardening took place, as described in the earlier paper. ${ }^{1}$

Magazine of Concrete Research, 2005, 57, No. 4 
Each image comprises 256 pixels horizontally in the $70 \mathrm{~mm}$ field-of-view, corresponding to an image pixel resolution of $273 \mu \mathrm{m}$. The same resolution was used in the vertical direction, but since the specimen's location was fixed and relatively small in the vertical direction, only 128 pixels were scanned to reduce the scanning time. Each reading was taken with a repetition time $\left(T_{\mathrm{R}}\right)$ of $100 \mathrm{~ms}$, with four readings being averaged, using the spin echo protocol. Sixteen slices, each $2 \cdot 19 \mathrm{~mm}$ thick, were taken through the thickness of the beam so that the whole beam was included. Each scan and the associated processing took about $30 \mathrm{~min}$. Since the concrete was about $3 \mathrm{~h}$ old when the scanning started it was $10 \mathrm{~h}$ old at the end.

The composite 2-D slice shown in Fig. 10 was taken from the 3-D data along the middle of the beam. The segregation in the mix is clearly visible, with very little aggregate in the top few millimetres. The AFRP shear links are clearly visible in the top as regularly spaced black dots. They are also visible near the bottom of the beam but are less easy to see in a single slice because they are difficult to distinguish from the aggregate. Each image shows slight distortion at the edges caused by the in-homogeneity of the gradient magnetic field. This is reflected in the apparent variation in the depth of the beam in the images and loss of precision at the edges.

The complete beam structure can be determined by identifying specific features (typically individual pieces of aggregate) that appear in the overlap region between two adjacent images. The individual images can then be superimposed and merged as shown in Fig. 11. In this way it is possible to produce an image of the structure of samples that are longer than the field of view of the MRI scanner.

The deflection as measured in the images, and the load as determined by the applied weights, were used to construct an approximate moment-deflection curve as shown in Fig. 12. The values are only approximate because creep of the concrete caused a reduction in load during the scanning procedure and the deflection in the image also reflects the stiffness of the reaction frame. Nevertheless, the shape of Fig. 12 is as expected, with an initially stiff response while the section is uncracked, followed by a reduction in stiffness after

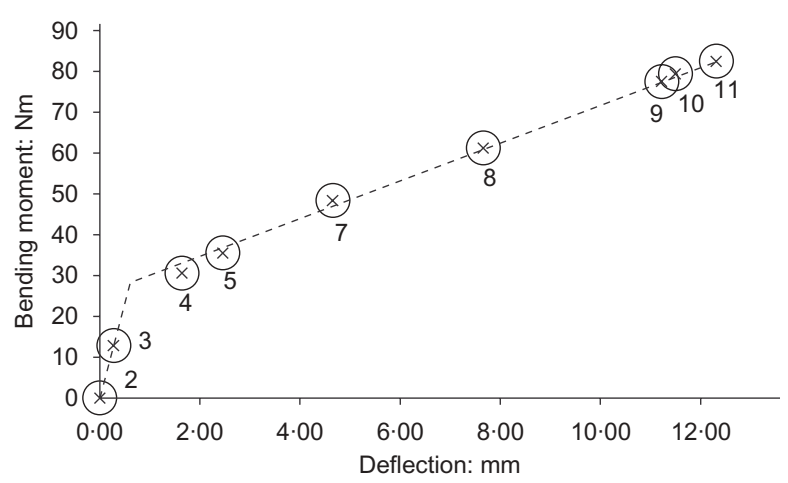

Fig. 12. Bending moment calculated using the pressure of the water in the loading system and deflection measured by counting pixels on the images. The numbers against each circle correspond to the loading stage when an MRI scan was performed
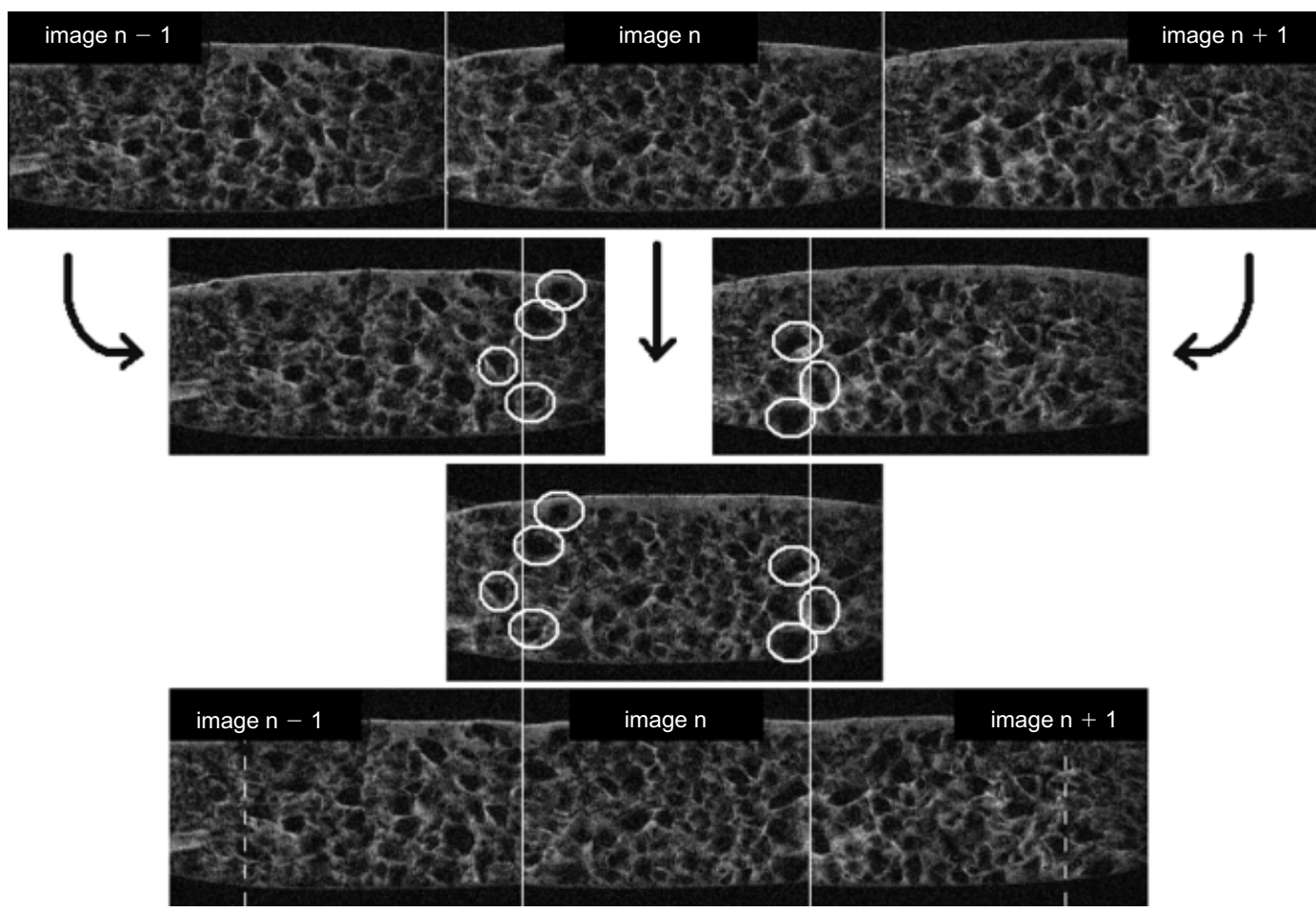

Fig. 11. Overlapping of structure images to reconstruct the whole sample 
cracking. Each circle on the figure corresponds to one scan of the beam.

The images from the successive 2-D scans are shown in Fig. 13. The image on the top left shows the central portion as obtained from the structure scan soon after casting, and the others show succeeding load stages. The image at the top right was obtained when the beam was unloaded and hence shows pre-existing fractures; there is one fairly large crack in the centre of the image and some small areas of whiteness are also visible below some larger pieces of aggregate. The background shows a very weak version of the structure image and there appears to be separation of the aggregate as it was placed around the shear links, reflected in the faint grey lines at each link position.

The bright white lines on the top and bottom of the beam and also at the bottom of the images, are from residual water on the surface of the beam and on the

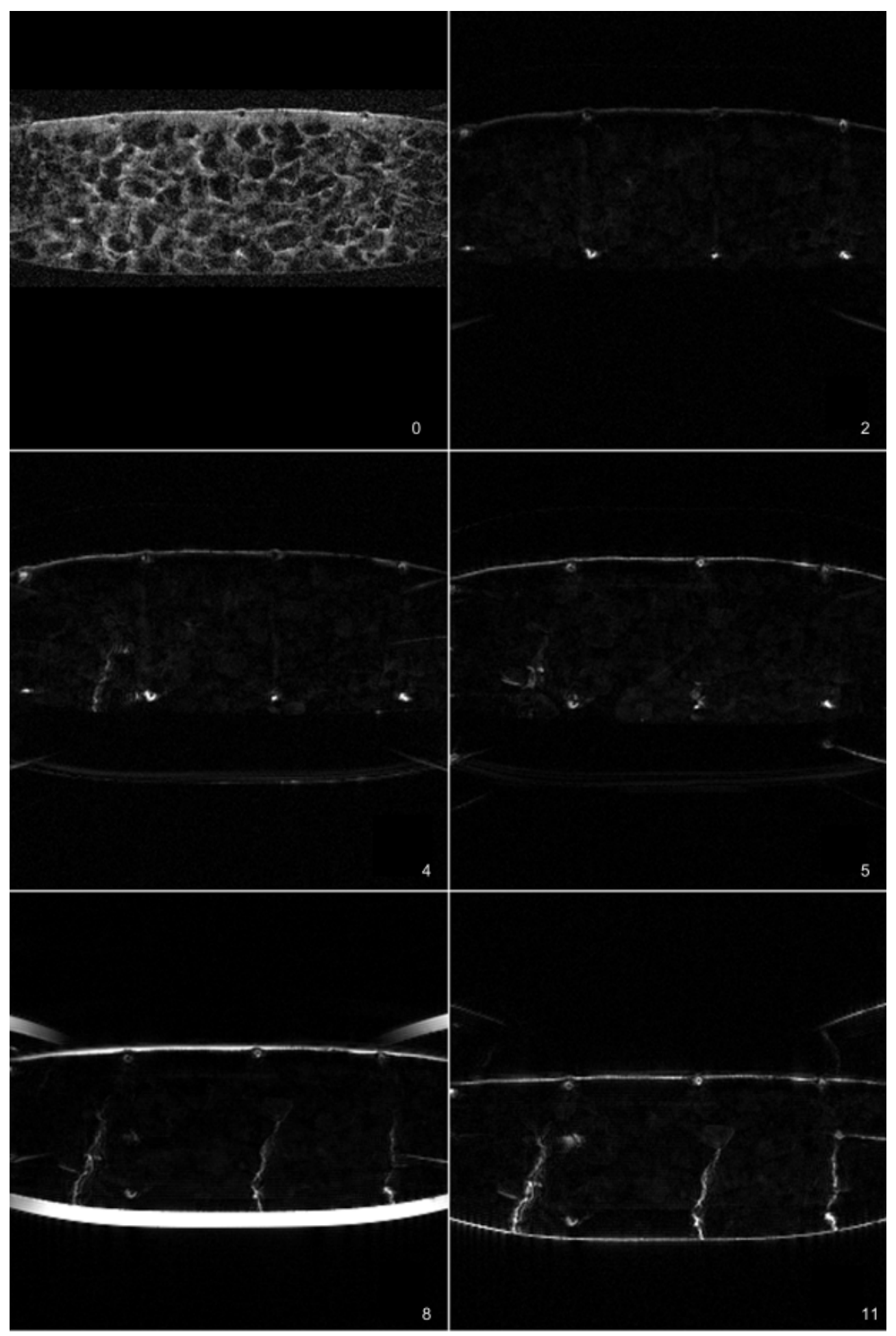

Fig. 13. Images obtained at successive loading stages. The image at the top left was obtained as part of the structure scan 
bottom of the tank. The deflection of the beam relative to the scanner is visible in the images and can be measured to obtain the deflection. The progress of cracking is also clear. The first significant crack is visible at load stage 4 , to the left of the image, and most significantly does not appear to start from the beam surface where the tensile stresses can be expected to be highest. By the next load stage it has propagated to the surface and it later extends. This reflects the unique information that can be obtained from a series of MRI scans.

Figure 14 shows a 3-D reconstruction of images which have been processed to remove all voxels that are darker than a certain threshold. This is effectively a 3-D map of the spaces inside the concrete that contain water. Pre-existing cracks around the shear reinforcement are clearly visible. Since the slice thickness was about ten times the in-plane pixel size, the vertical shear links and the longitudinal reinforcement are less clearly delineated. It remains to be determined whether this is due to the absence of cracks caused by the links being parallel to the casting direction, or if it is an artefact of the voxel dimensions. The surface characteristics of the axial reinforcement and the shear links may well differ because of the way they were made and the fact that they were made using different resins. These images clearly show the growth of the cracks from the bottom surface of the beam and they also show that some cracks form at the shear link positions, whereas others form between the shear links.

In this particular loading case there is no overall shear force in the beam within the field-of-view and, as expected, the cracks appear primarily as vertical surfaces extending to the neutral axis of the beam. However, local stress concentrations around aggregate and reinforcement can cause local stress variations, and this causes the cracks to diverge from the overall vertical alignment. This can be clearly seen in the expanded 2$\mathrm{D}$ slices shown in Fig. 15 of the region where one of the cracks forms away from the shear link. This crack appears to be diverted horizontally around a relatively large piece of aggregate and details of the fracture propagation can be clearly observed. These images have been co-registered spatially to eliminate the gross
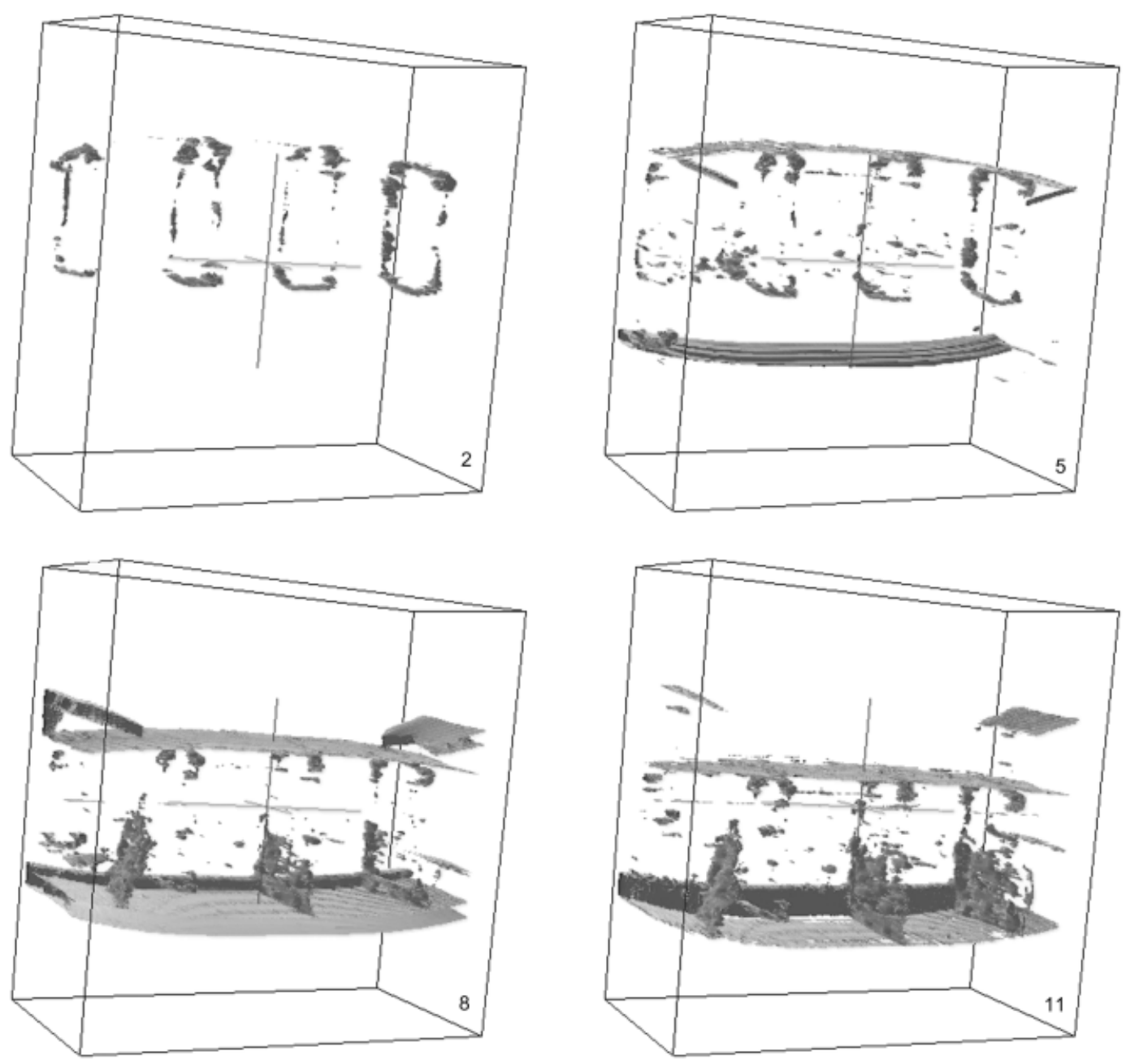

Fig. 14. Three-dimensional observation of the fracture at four different loading stages. In image 2, the water that has been detected is in the adhesive in the shear links; the later images show more water filling the expanding cracks. The images are shown inverted for clarity in publication-black shows the presence of water 


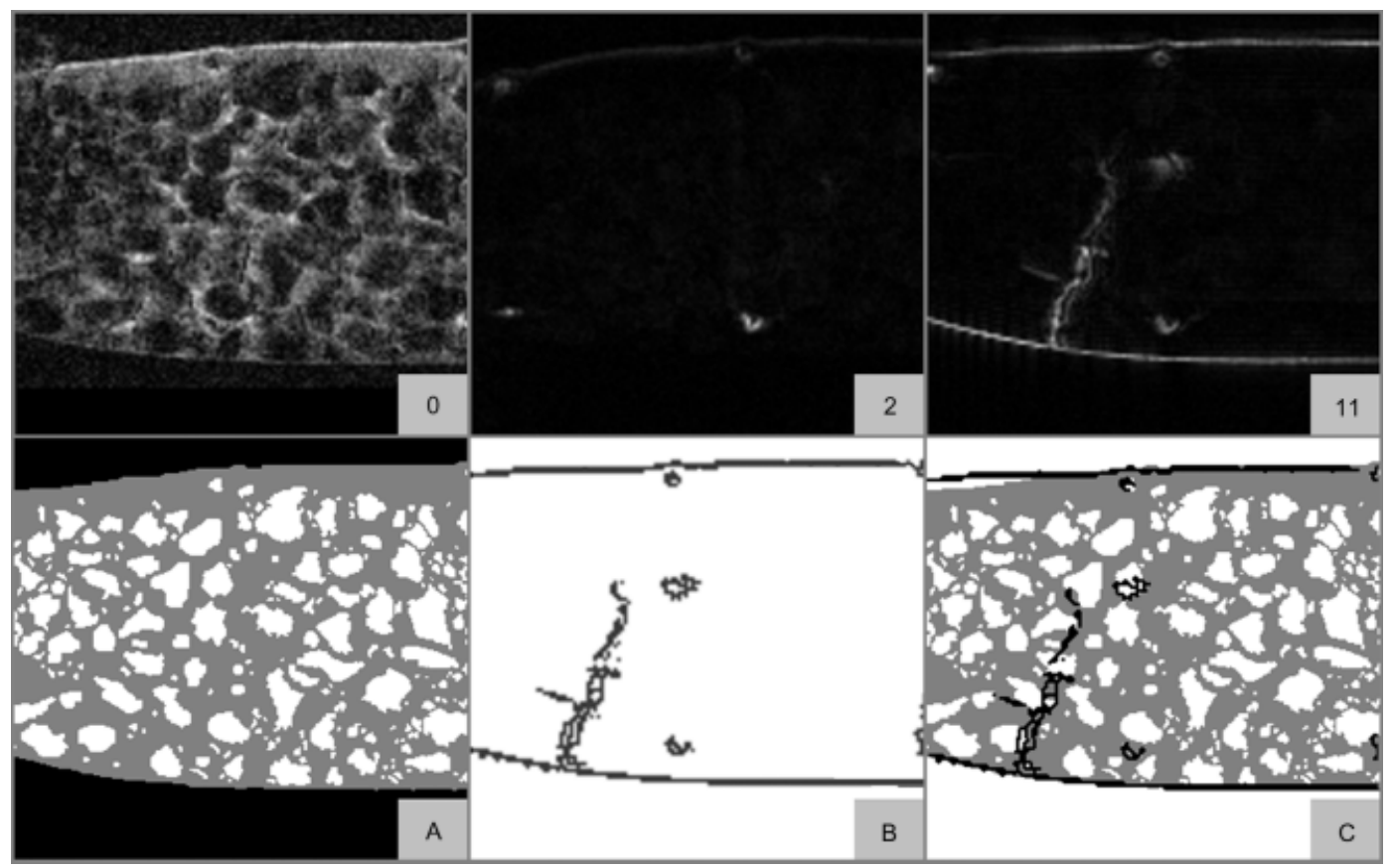

Fig. 15. Enlarged details of one crack at different loading stages. Images 0, 2 and 11 are details from the corresponding images in Fig. 13. Image A shows the aggregate as white and the matrix as grey from image 0; image B shows edge-detection applied to image 11; and image $C$ shows the two images superimposed. The relative positions of the aggregate and the crack are clearly seen, as are the two horizontal legs of one of the shear links. (These images are black and white for the purposes of publication. In normal use, image $B$ would be in false colour so that it can be merged directly with image 0)

movement caused by beam deflection, using the sections through the shear reinforcement as reference marks. The fracture image has been enhanced to eliminate the grey background.

It is accepted that there may be some influence of the pore water in the samples that are loaded underwater, or even when wet samples are tested in the air, since negative pressures can develop which will delay the initial crack opening. However, once the cracks have formed, the filling of cracks with water should not have a significant effect.

\section{Conclusion}

In conjunction with the previous two publications, this study has clearly demonstrated that MRI can be used to measure internal structures within a concrete beam and is also capable of determining the precise location of fractures within the concrete. It has been shown that, provided suitable spatial co-registration markers are available, it is possible to superimpose the structure and fracture images to show how the presence of individual pieces of aggregate can divert the cracks. Furthermore, this particular paper has demonstrated how a test frame can be assembled inside an MRI scanner to enable scans to be made while the specimen is under load. Since the testing method is non-destructive, it is possible to follow the progress of fracture as the loading increases.
To the best knowledge of the authors, MRI scanning is the only non-destructive technique for direct observation and quantitative measurement of internal crack structures in such detail, and it is believed that this paper and its companions are the first to demonstrate the procedures.

\section{Acknowledgments}

This work was supported by the EU TMR Network 'ConFibreCrete' and by the Herchel Smith Endowments

\section{References}

1. Marfisi E., Burgoyne C. J., Amin M. H. G. and Hall L. D. The use of MRI to observe the structure of concrete. Magazine of Concrete Research, 2005, 57, No. 2, 101-109.

2. Marfisi E., Burgoyne C. J., Amin M. H. G. and Hall L. D. The use of MRI to observe the fracture of concrete. Magazine of Concrete Research, 2005, 57, No. 2, 111-121.

3. Morphy R., Shehata E. and Rizkalla S. Bent effect on strength of CFRP stirrups. Proceedings of Fibre Reinforced Polymers for Reinforced Concrete Structures-3, Sapporo, 1997, 2, 19-26.

4. Ahmed E. A. Does core size affect strength testing? Concrete International, 1999, 21, 35-39.

Discussion contributions on this paper should reach the editor by 1 November 2005 